Document downloaded from:

http://hdl.handle.net/10251/50738

This paper must be cited as:

Muller, AA.; Sanabria Codesal, E.; Moldoveanu, A.; Asavei, V.; Soto Pacheco, P.; Boria Esbert, VE.; Lucyszyn, S. (2014). Apollonius unilateral transducer constant power gain circles on 3D Smith charts. Electronics Letters. 50(21):1531-1533. doi:10.1049/el.2014.2695.

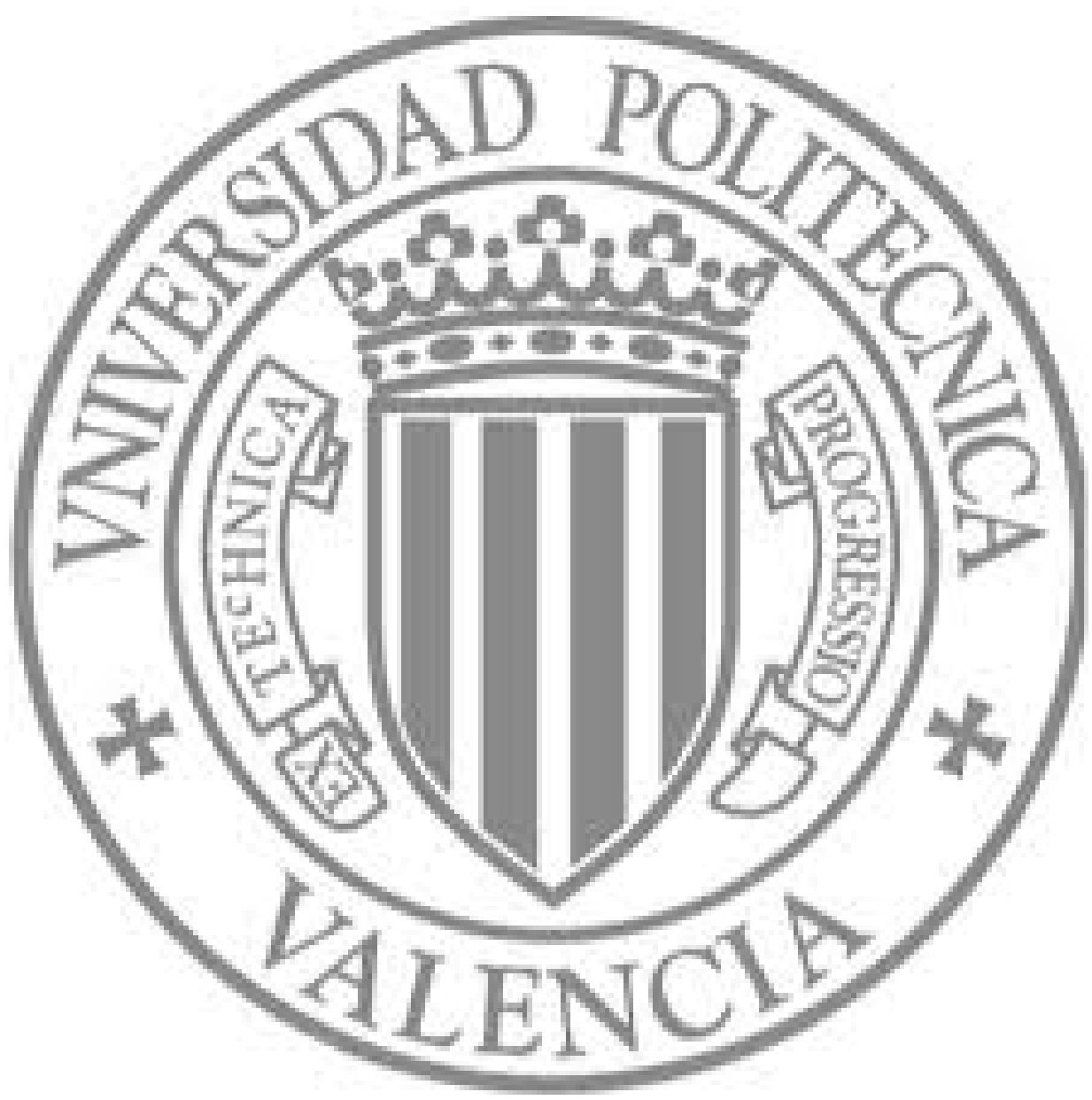

The final publication is available at

http://dx.doi.org/10.1049/el.2014.2695

Copyright Institution of Engineering and Technology (IET) 


\section{Apollonius Unilateral Transducer Constant Power Gain Circles on 3D Smith Charts}

\author{
A. A. Muller, E. Sanabria-Codesal, A. Moldoveanu, V. \\ Asavei, P. Soto, V. E. Boria, S. Lucyszyn
}

Unilateral transducer constant power gain circles play an essential role in the design of radio frequency amplifiers and active modulators, as they help to determine optimal impedance matching conditions to meet gain and stability specifications. In this article, it is shown that these gain circles are a subfamily of Apollonius circles. For better visualization, unilateral transducer constant gain power circles have been plotted for the first time on the 3D Smith chart. To this end, a natural relationship from inversive geometry was required, in order to relate the gain circles with cutting planes for the 3D Smith chart.

Introduction: In the design of radio frequency (RF) amplifiers and active modulators (e.g. frequency multipliers, mixers and small-shift frequency translators), the unilateral transducer constant power gain circles represent the loci of source and load impedances on a conventional 2-dimensional (2D) Smith chart [1]. Constant input and output impedance mismatch circles have also been recently introduced [2]; these are slightly different to gain circles, but share the same geometrical properties. Displaying power levels, or observing the Smith chart coverage [3] for a specific power level, leads to poor visualization when using $2 \mathrm{D}$ plots; gain and mismatch circles always converge to point circles (i.e., circles of zero radius) on the 2D Smith chart $[1,2]$.

Ellipses, hyperbolas and Apollonius circles are a set of points defined by two other points. On an ellipse, the sum of the distances to these two points is constant; while, on a hyperbola, the difference is constant. In the case of Apollonius circles, the ratio of the distances to these two points is constant [4], as seen in Fig 1.

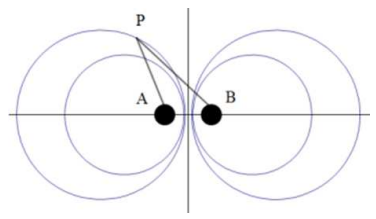

Fig. 1 Circles of Apollonius, with respect to points $A$ and $B: P A / P B$ ratio is constant for any point $P$ on the circle (2 reciprocal pairs of Apollonius circles are plotted). A and B are considered point circles, corresponding to a ratio of zero and infinity respectively [4]

The concept of an Apollonius gasket for microwave antenna design was recently introduced [5]. In this letter, we focus on the unilateral transducer constant power gain circle equations [1] and prove that they are a subfamily of Apollonius circles. As a result, the convergence of these gain circles to specific points on the Smith chart plane is not a matter of chance and, thus, it will be shown that the source and load impedances also share other geometrical properties.

Finally, the 3D Smith chart concept (based on the Riemann sphere) and associated Java tools, recently introduced by the authors [6, 7], are extended to plot gain circles using the unit sphere.

Gain circles on $2 D$ Smith chart: The unilateral transducer power gain for a 2-port network $G_{T U}$ is given by the textbook equations [1]:

$$
G_{T U}=G_{S} \cdot G_{O} \cdot G_{L} \quad ; \quad G(d B)=10 \cdot \log _{10}(G)
$$

where the respective intrinsic power gain factors associated with the source input stage, forward transmission of the transducer and load output stage are:

$$
G_{S}=\frac{1-\left|\Gamma_{S}\right|^{2}}{\left|1-S_{11} \cdot \Gamma_{S}\right|^{2}} ; G_{O}=\left|S_{21}\right|^{2} ; G_{L}=\frac{1-\left|\Gamma_{L}\right|^{2}}{\left|1-S_{22} \cdot \Gamma_{L}\right|^{2}}
$$

At the input stage, $\Gamma_{\mathrm{s}}$ is the voltage-wave reflection coefficient of the source impedance, while $S_{11}$ represents the input voltage-wave reflection coefficient of the 2-port network. Also, $S_{21}$ is the forward voltage-wave transmission coefficient of the 2-port network, while its reverse voltage-wave transmission coefficient $S_{12}$ is assumed to be zero. Finally, at the output stage, $\Gamma_{L}$ is the voltage-wave reflection coefficient of the load impedance, while $S_{22}$ represents the output voltage-wave reflection coefficient of the 2-port network. It should be noted that with purely passive stages/transducer the associated power gain factor $0 \leq G$ $\leq 1$ and $-\infty \leq G(d B) \leq 0$; while active circuits [8,9] can extend this range beyond unity to $0 \leq G \leq \infty$ and $-\infty \leq G(d B) \leq+\infty$.

For convenience, only the gain circles associated with input stage will be considered further, as the same approach applies to the output stage. Plotting $G_{S}$ gives the gain circles in the complex $\Gamma_{S}$-plane, contribution to the unilateral transducer power gain. As an example, consider a commercially available transistor (BFP 405 from Infineon Technologies AG), having measured values of magnitude and phase for $S_{11}$ of 0.707 and $-67^{\circ}$, respectively, at $1900 \mathrm{MHz}$ [1]. Fig. 2 shows the resulting family of source gain circles.
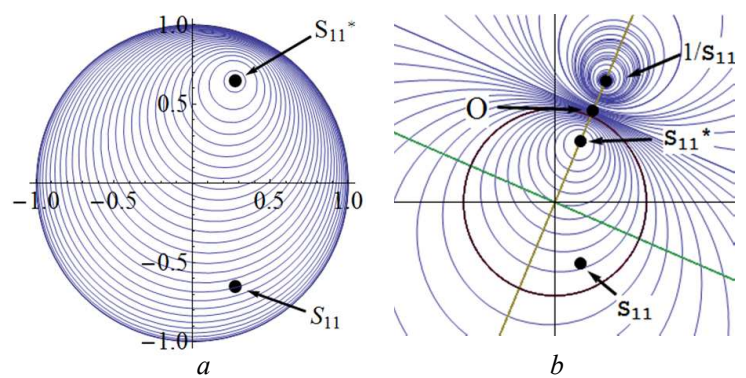

Fig. 2 Unilateral transducer constant power gain circles using (2), $\left(G_{O}=G_{L}=1\right.$ for illustration purposes) for $S_{11}=\left(0.707,-67^{\circ}\right)$ with: a $G_{S} \geq 0$ or $G_{s}(d B) \geq-\infty$

$b$ complete family $-\infty \leq G_{S} \leq+\infty$ (including the fictitious case $G_{S} \leq 0$ )

Gain circles associated with positive values for source input power gain factor $G_{S}$ are contained in the conventional 2D Smith chart, as seen in Fig. 2a. Note that, according to (2), the gain circle associated with $G_{S}$ $=0$ (i.e., $G_{S}(d B)=-\infty$ ) coincides with the Smith chart contour $\left|\Gamma_{S}\right|=1$. Moreover, positive $G_{S}$ gain circles converge to the point circle positioned at $S_{11}{ }^{*}$ (i.e., the complex conjugate of $S_{11}$ ) in the complex $\Gamma_{S^{-}}$ plane, which provides the maximum source input power gain factor [1]. However, after also plotting the negative $G_{S}$ gain circles, it is observed that there is convergence to the accumulation point $1 / S_{11}$, corresponding to $G_{\mathrm{s}}=-\infty$ (see Fig. 2b).

The equations for any point $P$ in the $\Gamma_{\mathrm{S}}$-plane moving along the Apollonius circles related to $A=S_{11}{ }^{*}$ and $B=1 / S_{11}$ are given from Fig. 1 by [4]:

$$
P A=m \cdot P B
$$

with $m>0$, or equivalently (considering the point $P=\Gamma_{S}$ ),

$$
\left|\Gamma_{\mathrm{s}}-S_{11}^{*}\right|^{2}=m^{2}\left|\Gamma_{\mathrm{s}}-1 / S_{11}\right|^{2}
$$

which, corresponds to the equation of a circle of radius $R$ and centre $C$ :

$$
R=\frac{m}{\left|m^{2}-1\right|}\left|S_{11}^{*}-1 / S_{11}\right| \quad ; \quad C=\frac{m^{2} / S_{11}-S_{11}^{*}}{m^{2}-1}
$$

The Apollonius circles derived here are now compared with the unilateral transducer constant power gain circles, whose radius and centre are [1]:

$$
R=\frac{\sqrt{1+\left(\left|S_{11}\right|^{2}-1\right) G_{\mathrm{s}}}}{\left.|1+| S_{11}\right|^{2} G_{\mathrm{s}} \mid} \quad ; \quad C=\frac{G_{\mathrm{s}} S_{11}^{*}}{1+G_{\mathrm{s}}\left|S_{11}\right|^{2}}
$$

Equating the expressions in (5) and (6) for the circle centres, the distance ratio $m$ can be expressed in terms of $G_{S}$ as:

$$
m=\left|S_{11}\right| \sqrt{1+G_{\mathrm{s}}\left(\left|S_{11}\right|^{2}-1\right)}
$$


It is important to note that (7) unifies the family of centre locations with the same radii and, thus, the families represented by (5) and (6) will coincide. In our particular case, points $A$ and $B$ are given by $(0.27625$, $0.65080)$ and $(0.55263,1.30199)$, respectively; the point $O$ is therefore $(0.41446,0.97639)$, which is placed in the infinite line obtained when the denominator of $R$ in (5) or (6) vanishes (i.e., where $m=1$ ). This gives the solution $G_{\mathrm{s}}=-2$ for the infinite line. The gain circles range from $G_{S}=0$ to $G_{S}=1 /\left(1-\left|\mathrm{S}_{11}\right|^{2}\right)=1.9994$ (i.e., between $-\infty \mathrm{dB}$ and +3 $\mathrm{dB})$. Considering (7), this means that $m$ varies between $\left|S_{I I}\right|$ and 0 and, thus, for any possible source impedance at point $P$, one can choose $A=$ $S_{11}{ }^{*}, B=1 / S_{11}$ and after varying $m$ between 0 and $\left|S_{11}\right|$ using (3) the entire family of unilateral (source) power gain circles can be obtained.

Gain circles on $3 D$ Smith chart: In order to exploit the recently proposed 3D Smith chart tool, a new representation for the power levels is given that overcomes the drawbacks associated with $2 \mathrm{D}$ visualizations $[1,3,10]$.

Starting with the example given in the previous section, if one wishes to express the unilateral transducer power gain associated with each gain circle defined by (2), the $0 \mathrm{~dB}$ unilateral transducer power gain will be found when the gain circle passes through the centre of the 2D Smith chart (north pole- on the 3D Smith chart); while in our example $\mathrm{a}+3 \mathrm{~dB}$ unilateral transducer power gain will be found for a gain circle having a centre point at $S_{11}{ }^{*}$. This can be seen in Fig. 3, while Fig. 4 shows the entire range of gain circles.

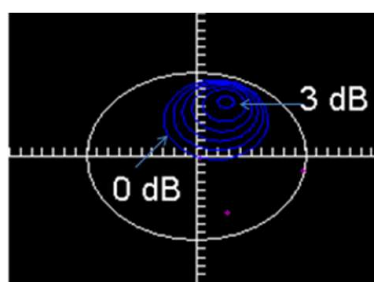

$a$

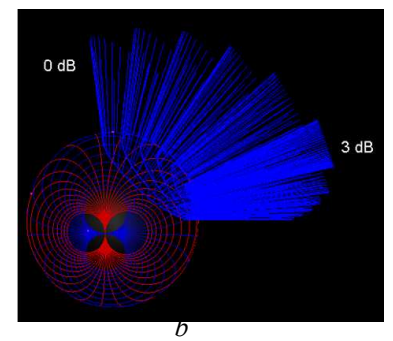

Fig. 3 Unilateral transducer constant power gain circles using (2) (with $G_{O}=G_{L}=1$ for illustration purposes) for $S_{11}=\left(0.707,-67^{\circ}\right), 0 \leq G_{S}(d B)$ $\leq+3$ (i.e., only part of the family): a $2 D$

$b 3 D$

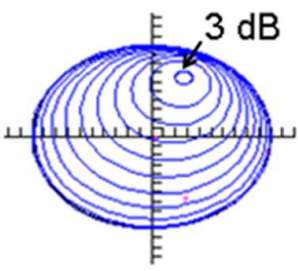

$a$

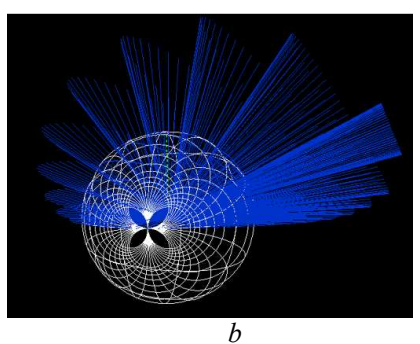

$b$
Fig. 4 Expanded view of Fig. 3 for $S_{11}=\left(0.707,-67^{\circ}\right),\left(-\infty \leq G_{S}(d B) \leq\right.$ $+3)$

a $2 D$

$b 3 D$

Mathematical observation: Since the gain circles are a subfamily of the Apollonius circles, it can be proven that while plotting these on the 3D Smith chart their cutting planes will always meet on the infinite line for each value of $S_{11}$ and, thus, on the axes of symmetry for the Apollonius circles. This can be seen in Fig. 5.

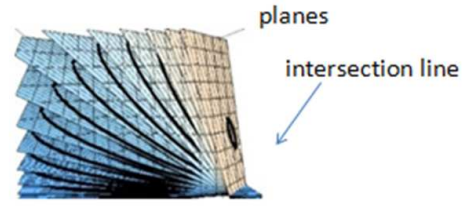

Fig. 5 Symmetry axes of the Apollonius circles when seen on the $3 D$ Smith chart, showing the gain circles' meeting line
Each gain circle corresponds to a cutting plane defined by (8), with $a$ and $b$ being the real and imaginary part of $S_{11}$, respectively:

$$
2(a x+b y) G_{\mathrm{s}}+\left(2+\left(\left|S_{11}\right|^{2}-1\right) G_{\mathrm{s}}\right) z=\left(1+\left|S_{11}\right|^{2}\right) G_{\mathrm{s}}
$$

Conclusion: It has been shown that unilateral transducer constant power gain circles (for the source input in our example) are a subfamily of the Apollonius circles, with respect to $S_{11}{ }^{*}$ and $1 / S_{11}$. These gain circles have been plotted on the 3D Smith chart for the first time, as a newly proposed visualization tool, overcoming traditional limits associated with contour plots on the traditional 2D Smith chart. It is interesting to note that unilateral transducer constant power gain circles are not the only Apollonius circles found in RF theory. Indeed, the concept described here can also be applied to the design of antennas [10] and provide mismatch and noise figure circles, among others.

Acknowledgments: This work has been partly funded by the FP7 PCIG11-2012-322162 Marie Curie CIG, POSDRU/159/1.5/S/134398 and by DGCYT grant MTM2012-33073.

Andrei A. Muller, Pablo Soto, Vicente E. Boria, (Microwave Applications Group, iTEAM, Universidad Politehnica de Valencia, Spain)

E-mail: andrei.stefan1@gmail.com

Esther Sanabria-Codesal, (Departamento de Matemática Aplicada, Universitat Politècnica de València, Spain)

Alin Moldoveanu, Victor Asavei, (Faculty of Automatic Control and Computers, University Politehnica Bucuresti, Romania)

Stepan Lucyszyn, (Department of Electrical and Electronic Engineering, Imperial College London, Exhibition Road, London, SW7 2AZ, UK)

\section{References}

1. Gilmore R., Besser L.: 'Practical RF circuit design for modern wireless systems - Active Circuits and Systems', vol. 2 (Artech House 2003)

2. Ciccognani W., Longhi P.E., Colangeli S., Limiti E.: 'Constant mismatch circles and application to low-noise microwave amplifier design', IEEE Transactions on Microwave Theory and Techniques, 2013, 61, no. 12, pp. 4154-4167

3. Sanchez P.C., Mingo. J, Ducar P.G., Carro. G., Valdovinos. A.: 'Figures of merit and performance measurements for RF and microwave tunable matching networks', European Microwave Integrated Circuits Conference, Manchester, 2011, pp. 402-405

4. Brannan D. A., Esplen M., Gray J.J.: 'Geometry' (Cambridge University Press, 2009)

5. Mukherjee B., Prgati P., Mukherjee J.: 'Hemispherical dielectric resonator antenna based on the Apollonian gasket of circles - A fractal approach', IEEE Transactions on Antennas and Propagation, 2014, 62 no. 1 , pp. $40-48$

6. Muller A.A., Soto. P, Dascalu D., Neculoiu D., Boria V.E.: 'A 3D Smith chart based on the Riemann sphere for active and passive microwave circuits', IEEE Microwave and Wireless Letters, 2011, 2, no. 6 , pp. $286-288$

7. Muller A.A., Soto P., Dascalu D., Boria V.E.: The 3D Smith chart and its practical applications', Microwave Journal, 2012. 5, no. 7, pp. 64-74

8. Lucyszyn S. and S, Robertson D.I.: 'Monolithic narrow band filter using ultrahigh-Q tunable active inductors', IEEE Transactions on Microwave Theory and Techniques, 42, 1994, pp. 2617-2622

9. Robertson D.I, Lucyszyn S.: 'RFIC and MMIC Design and Technology (IEE, London, UK, 2001)

10. Bario S.C., Bahrmazy P., Svendsen S., Jagielski O.: Pedersen G.F., 'Thermal loss in high-Q antennas', Electron Lett, 50, no. 13, 2014, pp 917-919 


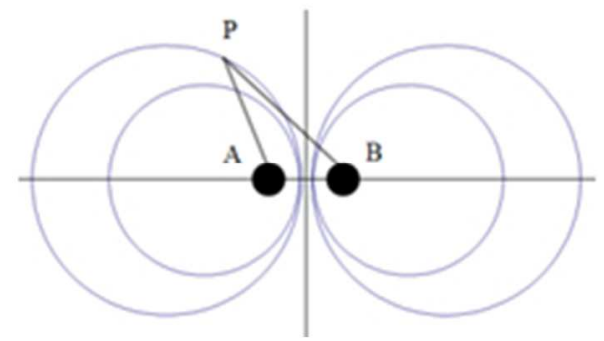

Fig. 1 Circles of Apollonius, with respect to points $A$ and $B$ : PA/PB ratio is constant for any point $P$ on the circle ( 2 reciprocal pairs of Apollonius circles are plotted). A and $B$ are considered point circles, corresponding to a ratio of zero and infinity respectively [4]. 

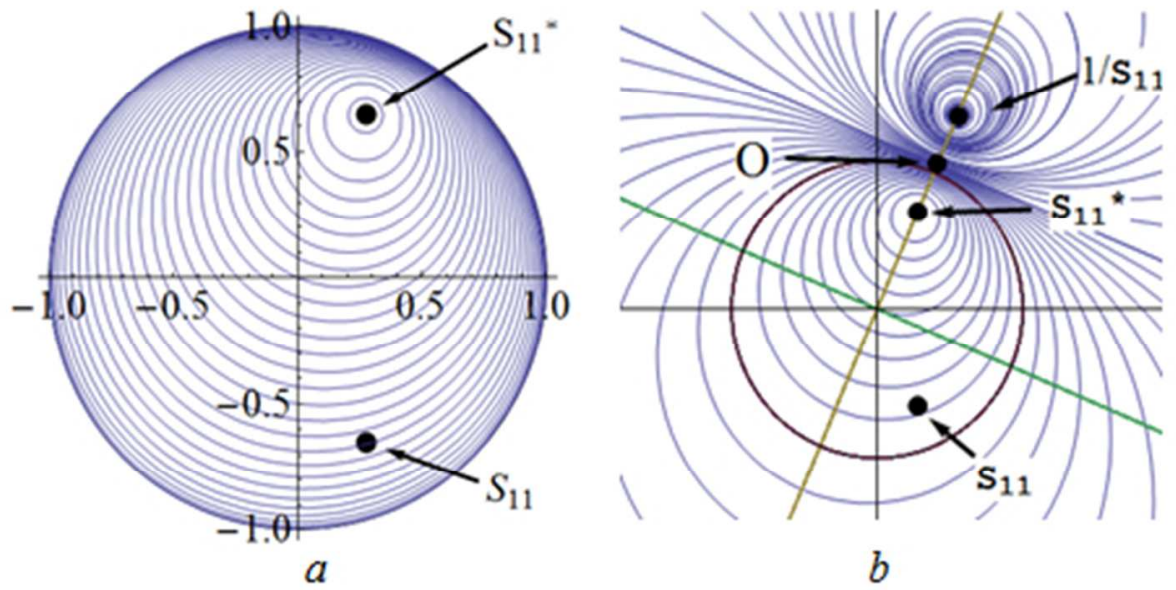

Fig. 2 Unilateral transducer constant power gain circles using (2), (GO=GL=1 for illustration purposes) for $\mathrm{S} 11=\left(0.707,-67^{\circ}\right)$ with:

a GS $\geq 0$ or $\mathrm{Gs}(\mathrm{dB}) \geq-\infty$

b complete family $-\infty \leq$ GS $\leq+\infty$ (including the fictitious case GS $\leq 0$ ) 


\section{Page 5 of 7}
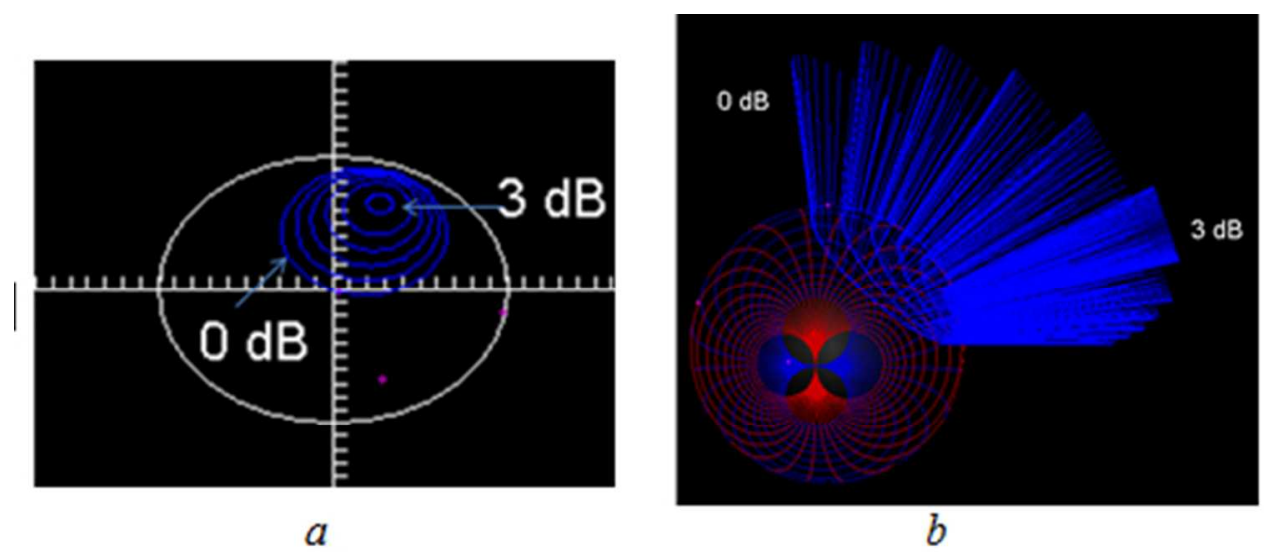

Fig. 3 Unilateral transducer constant power gain circles using (2) (with $\mathrm{GO}=\mathrm{GL}=1$ for illustration purposes) for $\mathrm{S} 11=\left(0.707,-67^{\circ}\right), 0 \leq \mathrm{GS}(\mathrm{dB}) \leq+3$ (i.e., only part of the family):

$$
\text { a 2D }
$$



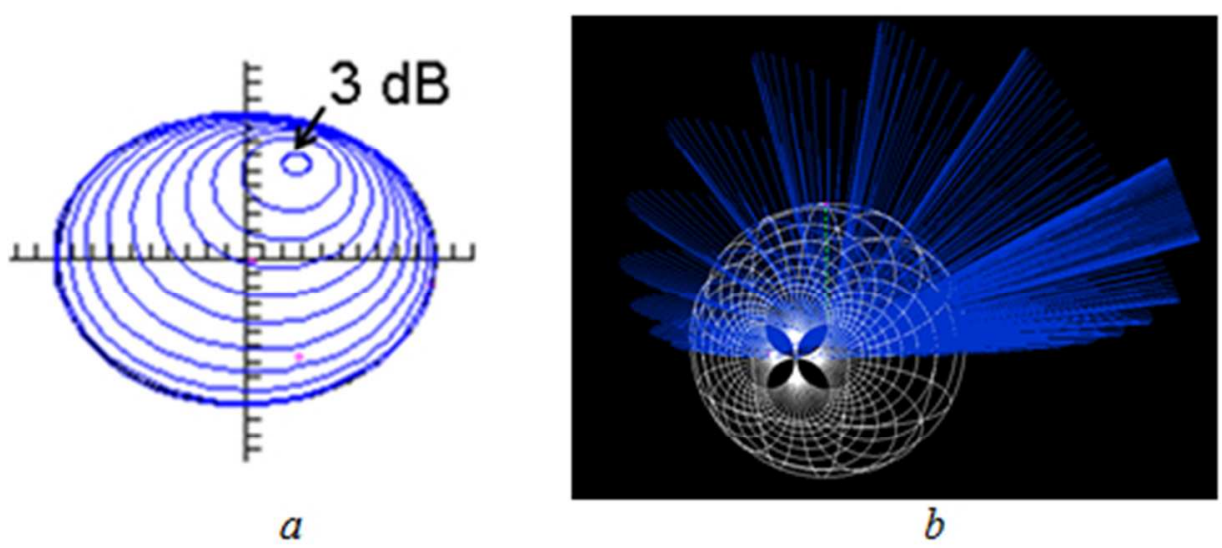

Fig. 4 Expanded view of Fig. 3 for $\mathrm{S} 11=\left(0.707,-67^{\circ}\right),(-\infty \leq \mathrm{GS}(\mathrm{dB}) \leq+3)$ a 2D
b 3D 


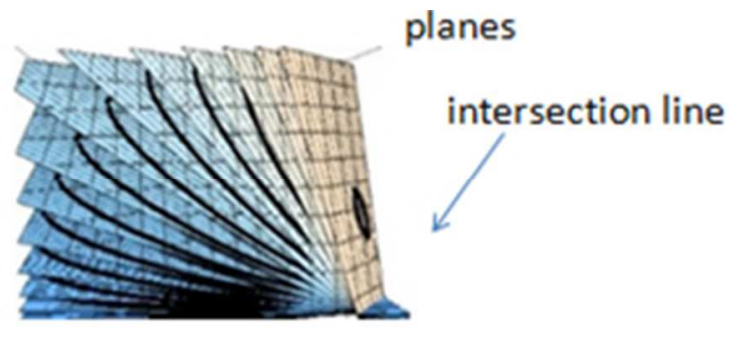

Fig. 5 Symmetry axes of the Apollonius circles when seen on the 3D Smith chart, showing the gain circles' meeting line 